Provided for non-commercial research and education use. Not for reproduction, distribution or commercial use.

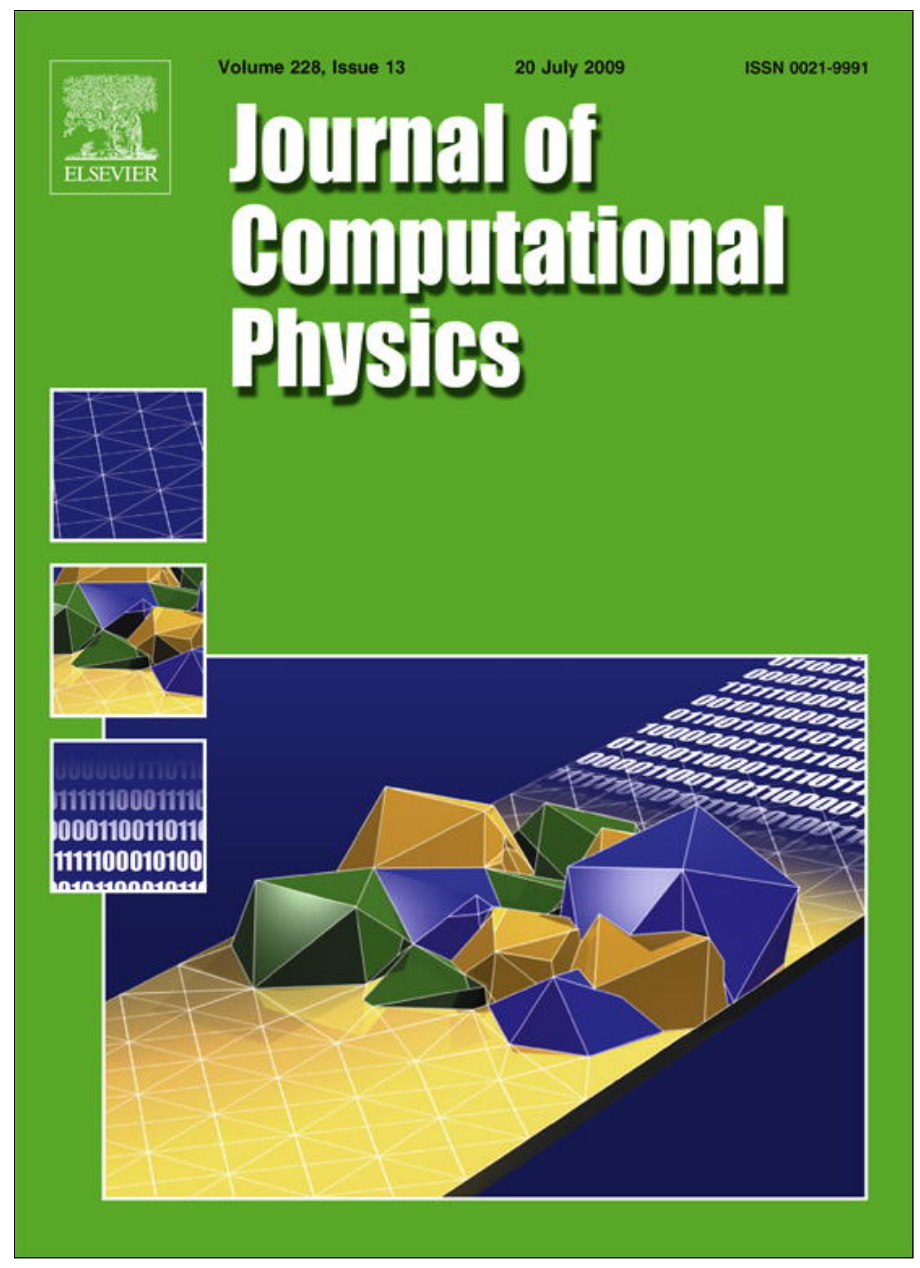

This article appeared in a journal published by Elsevier. The attached copy is furnished to the author for internal non-commercial research and education use, including for instruction at the authors institution and sharing with colleagues.

Other uses, including reproduction and distribution, or selling or licensing copies, or posting to personal, institutional or third party websites are prohibited.

In most cases authors are permitted to post their version of the article (e.g. in Word or Tex form) to their personal website or institutional repository. Authors requiring further information regarding Elsevier's archiving and manuscript policies are encouraged to visit:

http://www.elsevier.com/copyright 


\title{
BECOOL: Ballooning eigensolver with COOL finite elements
}

\author{
G.A. Cooper ${ }^{\mathrm{a}, *}$, J.P. Graves ${ }^{\mathrm{b}}$, W.A. Cooper ${ }^{\mathrm{b}}$, R. Gruber ${ }^{\mathrm{c}}$, R.S. Peterson ${ }^{\mathrm{a}}$ \\ a Physics Department, University of the South, Sewanee, Tennessee 37383, United States \\ ${ }^{\mathrm{b}}$ Ecole Polytechnique Fédérale de Lausanne, Centre de Recherches en Physique des Plasmas, Association Euratom-Suisse, CH1015 Lausanne, Switzerland \\ ${ }^{\mathrm{c}}$ Ecole Polytechnique Fédérale de Lausanne, Laboratoire D'Ingénierie Numérique, CH1015 Lausanne, Switzerland
}

\section{A R T I C L E I N F O}

\section{Article history:}

Received 29 September 2008

Received in revised form 20 February 2009

Accepted 5 April 2009

Available online 16 April 2009

\section{Keywords:}

Ballooning modes

Variational formulation

COOL finite elements

Block pentadiagonal matrix

Inverse vector iteration

\begin{abstract}
A B S T R A C T
An incompressible variational ideal ballooning mode equation is discretized with the COOL finite element discretization scheme using basis functions composed of variable order Legendre polynomials. This reduces the second order ordinary differential equation to a special block pentadiagonal matrix equation that is solved using an inverse vector iteration method. A benchmark test of BECOOL (Ballooning Eigensolver using COOL finite elements) with second order Legendre polynomials recovers precisely the eigenvalues computed by the VVBAL shooting code. Timing runs reveal the need to determine an optimal lower order case. Eigenvalue convergence runs show that cubic Legendre polynomials construct the optimal ballooning mode equation for intensive computations.
\end{abstract}

(c) 2009 Elsevier Inc. All rights reserved.

\section{Introduction}

The interaction of pressure gradients with magnetic field line curvature results in local magnetohydrodynamic instabilities referred to as ballooning modes because they tend to concentrate in regions with concave magnetic field line curvature. The study of these instabilities can yield important information about the limits that can be achieved by pressure gradients in magnetic confinement systems. The original theory was developed for axisymmetric tokamaks and reconciled periodicity with extended structures along the magnetic field lines in the presence of magnetic shear [1]. Ballooning mode equations in three dimensional geometry were derived by Dewar and Glasser [2] and independently by Correa-Restrepo [3]. These were fourth order ordinary differential equations that reduced to second order when ignoring compressibility.

The JMC code [4] developed and integrated the equation along a field line. Instability is indicated by the eigenfunction crossing zero, while stability is given when it diverges from zero. If the eigenfunction converges towards zero at a large distance along the field line, then the field line is marginally stable. The most common eigensolvers (VVBAL [5,6], 3D BALLOON [7], Nakajima code [8]) use a shooting mechanism in which the eigenvalue is adjusted until a continuous converged solution is computed. The most advanced ballooning solver at the moment is COBRA [9] which solves the variational form of the ballooning equation using a centered finite difference scheme to convert the equation into a matrix eigenvalue problem. An inverse vector iteration method is used to converge an eigenvalue.

BECOOL applies the COOL (COnstraints Oriented Library) finite element discretization scheme [10,11] to the variational form of the ballooning equation. This uses Legendre polynomials of variable order for the construction of the basis functions with which to approximate the ballooning perturbation. The equation is then reduced to a special block pentadiagonal

\footnotetext{
* Corresponding author.

E-mail address: coopega0@sewanee.edu (G.A. Cooper).
} 
matrix eigenvalue problem. The PAMERA [12] routine solves the matrix equation through an inverse vector iteration method. PAMERA has been used successfully in the TERPSICHORE code [13] to investigate global magnetohydrodynamic stability. A ballooning equation where the kinetic energy has been replaced by the instability driving term multiplied by the eigenvalue is used in BECOOL. The sign of the eigenvalue determines whether the ballooning modes are stable or unstable while the amplitude of the eigenvalue yields a rough measure of how much the local pressure gradient must be amplified or diminished to obtain marginal stability (very near marginality it is almost an exact measure). For ballooning modes, the eigenvalue typically represents a growth rate of instability (or finite oscillation frequency for stability), however BECOOL does not generate an actual growth rate but instead a simple check of stability or instability. The ballooning mode equation is discretized in Section 2. In Section 3, the discretized equation is reduced to a special block pentadiagonal matrix equation whose individual block equations are derived. Numerical tests are made in Sections 4 and 5 contains the summary and conclusions.

\section{COOL discretization [10]}

The second order ordinary differential equation that models ballooning modes assumes the form [6],

$$
\int_{-\infty}^{\infty} d \theta\left\{\left[C_{p}+C_{s}\left(\theta-\theta_{k}\right)+C_{q}\left(\theta-\theta_{k}\right)^{2}\right]\left(\frac{\partial Y}{\partial \theta}\right)^{2}-\left[d_{p}+d_{s}\left(\theta-\theta_{k}\right)\right] Y^{2}(\theta)\right\}=\lambda \int_{-\infty}^{\infty} d \theta\left[d_{p}+d_{s}\left(\theta-\theta_{k}\right)\right] Y^{2}(\theta)
$$

where $\theta$ is the poloidal angle, $\theta_{k}$ is the radial wave number, $Y$ is the perturbation variable and $\lambda$ is the eigenvalue. In the COOL discretization scheme, this equation is broken into $N$ intervals. The variable $\theta$ is replaced by the index $J$ that labels the interval and a variable, $-1<r<1$, which specifies the location within the interval where $r=0$ is the center of the interval. The infinite integral thus becomes a summation over $J$ of integrals over $r$ from -1 to 1 . The following equation relates $\theta$ to $r$ :

$$
\theta=\theta_{J-1 / 2}+\frac{\Delta \theta}{2} r
$$

where $\Delta \theta$ is the length of the interval in radians. The perturbation variable, $Y$, is approximated by a series of basis functions composed of variable order Legendre polynomials.

$$
Y(r)=Y_{J-1} h_{1}(r)+\sum_{i=2}^{p} Y_{J-1 / 2}^{i} h_{i}(r)+Y_{J} h_{p+1}(r)
$$

$Y_{J-1}$ and $Y_{J}$ corresponds to the unknown perturbation function at the beginning point and end point of the interval. The $Y_{J-1 / 2}^{i}$ elements correspond to the unknown perturbation function evaluated at the Gauss-points of the Legendre polynomial along the interval. The choice we make for the basis functions is strongly motivated by the requirement that the divergence of the perturbed displacement vector vanish in incompressible linear magnetohydrodynamics. If this is satisfied, the modes can be decoupled and spectral pollution is avoided [14]. This issue is particularly important near marginal stability. The Legendre polynomial basis function model we have applied complies exactly with this condition [10,11]. The pollution-free nonconforming numerical approach proposed in Ref. [14] was limited only to polynomials of order $p=0$ and 1. The COOL method generalizes this approach to higher order polynomials $p>1$. The $h$ basis functions are computed as follows:

$$
h_{i}(r)=\beta_{i} \frac{\left(1-r^{2}\right) L_{p}(r)}{\left(r-\zeta_{1}\right)\left(r-r_{i}\right)}
$$

where $L_{p}$ is a Legendre polynomial of variable order $p, \zeta_{1}$ is the value of the variable $r$ at a Gauss point of the Legendre polynomial (typically at the first zero). The index, $i$, varies from 1 to $p+1, r_{i}$ is -1 at $i=1,+1$ at $p+1$ and ranges from $\zeta_{2}$ to $\zeta_{p}$ as $i$ increments from 2 to $p$ while skipping one Gauss point (usually the first one). $\beta_{i}$ is a normalization factor that makes the value of $h_{i}$ equal to unity at its maximum. Furthermore, the following substitutions to the equation are made:

$$
\begin{aligned}
& \mathcal{A}(r)=\mathcal{A}(\theta)=C_{p}+C_{s}\left(\theta-\theta_{k}\right)+C_{q}\left(\theta-\theta_{k}\right)^{2} \\
& \mathcal{D}(r)=\mathcal{D}(\theta)=d_{p}+d_{s}\left(\theta-\theta_{k}\right)
\end{aligned}
$$

$\mathcal{A}(r)$ is the stabilizing field line bending term and $\mathcal{D}(r)$ is the interaction of the pressure gradient with the magnetic field line curvature. $\mathcal{D}(r)$, the driving term of instability, is used as a scale factor for modeling the kinetic energy. This choice displaces the continuous spectrum away from the marginal point allowing for discrete eigenvalues in the stable domain. The discretized ballooning mode equation thus adopts the following form:

$$
\sum_{J=1}^{N} \frac{\Delta \theta}{2} \int_{-1}^{1} d r\left\{\frac{\partial Y}{\partial r}\left(\frac{2}{\Delta \theta}\right)^{2} \mathcal{A}(r) \frac{\partial Y}{\partial r}-Y(r) \mathcal{D}(r) Y(r)=\lambda Y(r) \mathcal{D}(r) Y(r)\right\}
$$

The infinite domain of integration has been truncated into a finite domain of $N$ intervals. It has to be verified a posteriori that the extent of the truncated domain along the magnetic field line is adequate to resolve a well converged ballooning eigenfunction (as shown later in Fig. 5). The integral is then solved using the Gauss-Legendre quadrature rule [15]. 


\section{Matrix construction}

By substituting Eq. (3) into the equation that results from solving Eq. (7) with the Gauss-Legendre quadrature rule and separating out the terms according to their associated perturbation functions, we arrive in the variationally weak form at a special block pentadiagonal matrix problem given by $\mathbf{A x}=\lambda \mathbf{B x}$. Each interval of the matrix $\mathbf{A}$ is composed of a matrix that contains four corner sub-blocks (A, BL, BU, C) of dimension $1 \times 1$, two outer row blocks $(\mathrm{E}, \mathrm{H})$ of dimension $1 \times(p-1)$, two outer column blocks $(\mathrm{F}, \mathrm{G})$ of dimension $(p-1) \times 1$ and a central matrix (D) of dimension $(p-1) \times(p-1)$. The upper left corner block $(\mathrm{BU})$ of each matrix after the first interval is replaced by itself plus the lower right corner $(\mathrm{BL})$ of the matrix of the preceding interval $(B(J)=B U(J)+B L(J-1)$, see Fig. 1). The matrix B is similarly constructed with sub-blocks BA, $\mathrm{BBL}, \mathrm{BBU}, \mathrm{BC}, \mathrm{BE}, \mathrm{BH}, \mathrm{BF}, \mathrm{BG}$ and BD. Boundary conditions are implemented to guarantee a vanishing of the eigenfunction at the ends of the field line. The following are the equations for the sub-blocks of the $J$ interval corresponding to the $\mathbf{A}$ and $\mathbf{B}$ matrices:

$$
\begin{aligned}
& B U(J)=\frac{\Delta \theta}{2} \sum_{m=1}^{p} w_{m}\left[\left.\left.\frac{d h_{1}}{d r}\right|_{r=\zeta_{m}} \mathcal{A}\left(S_{m}\right)\left(\frac{2}{\Delta \theta}\right)^{2} \frac{d h_{1}}{d r}\right|_{r=\zeta_{m}}-h_{1}\left(\zeta_{m}\right) \mathcal{D}\left(S_{m}\right) h_{1}\left(\zeta_{m}\right)\right] \\
& B L(J)=\frac{\Delta \theta}{2} \sum_{m=1}^{p} w_{m}\left[\left.\left.\frac{d h_{p+1}}{d r}\right|_{r=\zeta_{m}} \mathcal{A}\left(S_{m}\right)\left(\frac{2}{\Delta \theta}\right)^{2} \frac{d h_{p+1}}{d r}\right|_{r=\zeta_{m}}-h_{p+1}\left(\zeta_{m}\right) \mathcal{D}\left(S_{m}\right) h_{p+1}\left(\zeta_{m}\right)\right] \\
& C(J)=\frac{\Delta \theta}{2} \sum_{m=1}^{p} w_{m}\left[\left.\left.\frac{d h_{1}}{d r}\right|_{r=\zeta_{m}} \mathcal{A}\left(S_{m}\right)\left(\frac{2}{\Delta \theta}\right)^{2} \frac{d h_{p+1}}{d r}\right|_{r=\zeta_{m}}-h_{1}\left(\zeta_{m}\right) \mathcal{D}\left(S_{m}\right) h_{p+1}\left(\zeta_{m}\right)\right] \\
& A(J)=C(J) \\
& E_{i-1}(J)=\frac{\Delta \theta}{2} \sum_{m=1}^{p} w_{m}\left[\left.\left.\frac{d h_{1}}{d r}\right|_{r=\zeta_{m}} \mathcal{A}\left(S_{m}\right)\left(\frac{2}{\Delta \theta}\right)^{2} \frac{d h_{i}}{d r}\right|_{r=\zeta_{m}}-h_{1}\left(\zeta_{m}\right) \mathcal{D}\left(S_{m}\right) h_{i}\left(\zeta_{m}\right)\right] \\
& H_{i-1}(J)=\frac{\Delta \theta}{2} \sum_{m=1}^{p} w_{m}\left[\left.\left.\frac{d h_{i}}{d r}\right|_{r=\zeta_{m}} \mathcal{A}\left(S_{m}\right)\left(\frac{2}{\Delta \theta}\right)^{2} \frac{d h_{p+1}}{d r}\right|_{r=\zeta_{m}}-h_{i}\left(\zeta_{m}\right) \mathcal{D}\left(S_{m}\right) h_{p+1}\left(\zeta_{m}\right)\right] \\
& F_{i-1}(J)=E_{i-1}(J) \\
& G_{i-1}(J)=H_{i-1}(J) \\
& D_{i-1, k-1}(J)=\frac{\Delta \theta}{2} \sum_{m=1}^{p} w_{m}\left[\left.\left.\frac{d h_{i}}{d r}\right|_{r=\zeta_{m}} \mathcal{A}\left(S_{m}\right)\left(\frac{2}{\Delta \theta}\right)^{2} \frac{d h_{k}}{d r}\right|_{r=\zeta_{m}}-h_{i}\left(\zeta_{m}\right) \mathcal{D}\left(S_{m}\right) h_{k}\left(\zeta_{m}\right)\right] \\
& B B U(J)=\frac{\Delta \theta}{2} \sum_{m=1}^{p} w_{m} h_{1}\left(\zeta_{m}\right) \mathcal{D}\left(S_{m}\right) h_{1}\left(\zeta_{m}\right) \\
& B B L(J)=\frac{\Delta \theta}{2} \sum_{m=1}^{p} w_{m} h_{p+1}\left(\zeta_{m}\right) \mathcal{D}\left(S_{m}\right) h_{p+1}\left(\zeta_{m}\right) \\
& B C(J)=\frac{\Delta \theta}{2} \sum_{m=1}^{p} w_{m} h_{1}\left(\zeta_{m}\right) \mathcal{D}\left(S_{m}\right) h_{p+1}\left(\zeta_{m}\right) \\
& B A(J)=B C(J) \\
& B E_{i-1}(J)=\frac{\Delta \theta}{2} \sum_{m=1}^{p} w_{m} h_{1}\left(\zeta_{m}\right) \mathcal{D}\left(S_{m}\right) h_{i}\left(\zeta_{m}\right) \\
& B H_{i-1}(J)=\frac{\Delta \theta}{2} \sum_{m=1}^{p} w_{m} h_{i}\left(\zeta_{m}\right) \mathcal{D}\left(S_{m}\right) h_{p+1}\left(\zeta_{m}\right) \\
& B F_{i-1}(J)=B E_{i-1}(J) \\
& B G_{i-1}(J)=B H_{i-1}(J) \\
& B D_{i-1, k-1}(J)=\frac{\Delta \theta}{2} \sum_{m=1}^{p} w_{m} h_{i}\left(\zeta_{m}\right) \mathcal{D}\left(S_{m}\right) h_{k}\left(\zeta_{m}\right)
\end{aligned}
$$

$\zeta_{m}$ is a value that ranges from -1 to 1 and locates a Gauss point of the Legendre polynomial indexed by $m$ within the interval. $w_{m}$ is the weight of the Gauss-Legendre quadrature rule that corresponds to the Gauss point, $\zeta_{m}$, within each interval and the $i$ and $k$ are indexes that range from 2 to p. $S_{m}$ is a measure of the locations of the Gauss-points of each interval along the field line $\left(S_{m}=\theta_{J-1 / 2}+\frac{\Delta \theta}{2} \zeta_{m}\right)$. 


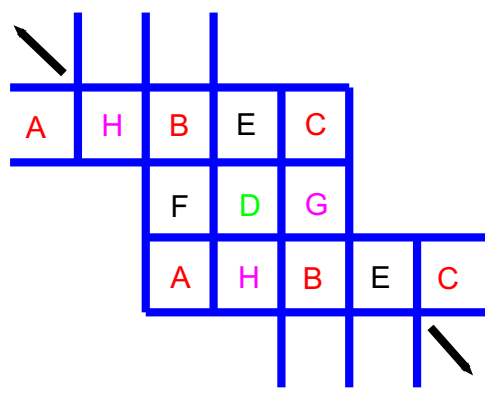

Fig. 1. Special block pentadiagonal matrix structure.

The matrix eigenvalue problem $\mathbf{A x}=\lambda \mathbf{B x}$ is solved with an inverse vector iteration technique because such an approach is very efficient, stable and, taking advantage of the special block pentadiagonal structure of the system, consumes only a small fraction of the computational effort which is dominated by the matrix construction. A detailed description of the PAMERA routine that implements the inverse vector iteration scheme is given in Ref. [13].

\section{Numerical computations}

The equilibrium analyzed and calculated with the VMEC code [16] corresponds to a ten period Heliotron case that models the Large Helical Device (LHD) in operation in Japan. This equilibrium has been used extensively for benchmark studies of stellarator codes [17]. The equilibrium state undergoes a transformation to the Boozer coordinate reference frame [18] through the TERPSICHORE code which calculates the Fourier coefficients of $C_{p}, C_{s}, C_{q}, d_{p}$ and $d_{s}$ all found in Eqs. (5) and (6). A benchmark test was done with second order Legendre polynomials as a function of the radial position, $s$, within the plasma. This recovers the ballooning eigenvalues computed using the shooting mechanism in VVBAL (see Fig. 2). This test was calculated using 15 poloidal turns of the magnetic field line on all flux surfaces while ensuing computations employed 41 poloidal turns running on the flux surface $s=0.526$. We specifically choose the field line that passes through the region with the most destabilizing curvature. We select the radial wave number $\theta_{k}=0$ for all our tests as well as a convergence criterion of $1 \times 10^{-9}$. The convergence criterion affects the inverse vector iteration process as it has to satisfy this condition at any point along the magnetic field line from one iteration to the next. Once the criterion is satisfied, the eigenfunction and eigenvalue are considered to have converged. Timing tests for the variational ballooning equations with Legendre polynomials of differing order were run as a function of the number of intervals (see Fig. 3) where $h$ is the inverse of the number of intervals (not the be confused with the basis function which are labeled as $h_{i}$ ). It was found that the time taken to compute an eigenvalue increased exponentially as $p$ is incremented. As a result, it was determined that a small value of $p$ that converges rapidly is desired. Tests for convergence as a function of $h$ with differing orders were run to determine the optimal order as shown in Fig. 4. We found that the $p=3$ case converged more rapidly and with a less pronounced oscillation than the cases $p=2, p=4$ and $p=5$. We had anticipated that by increasing the order $p$, we could correspondingly reduce the number of intervals and still obtain converged eigenvalues. Unfortunately, this has proved not to be the case. It is to be noted

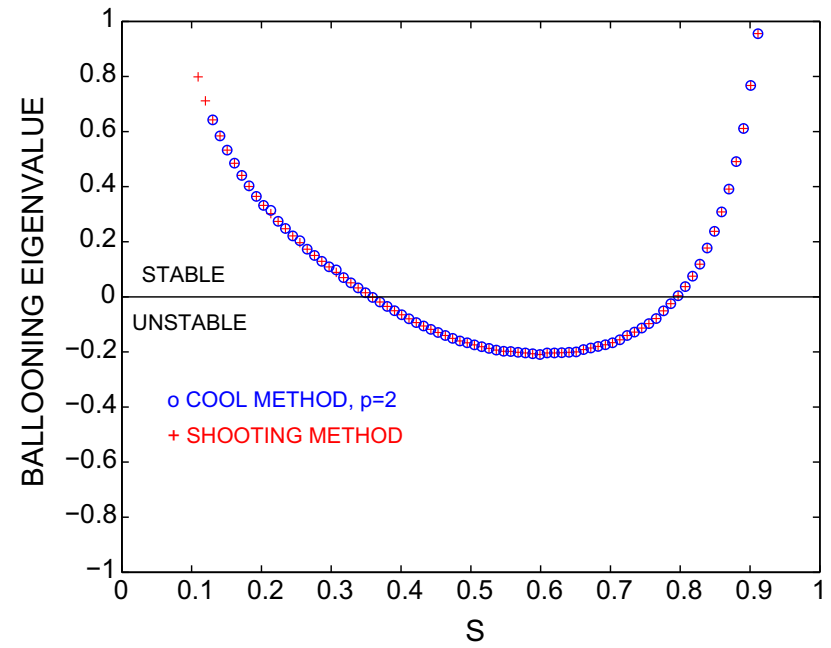

Fig. 2. Ballooning benchmark test as a function of the variable $s$, demonstrating that the variational CoOL scheme exactly recovers the eigenvalues obtained using the VVBAL shooting method. The run time of the COOL method computation was $\sim 29 s$, slightly more than 3 times faster than the shooting code benchmark. 


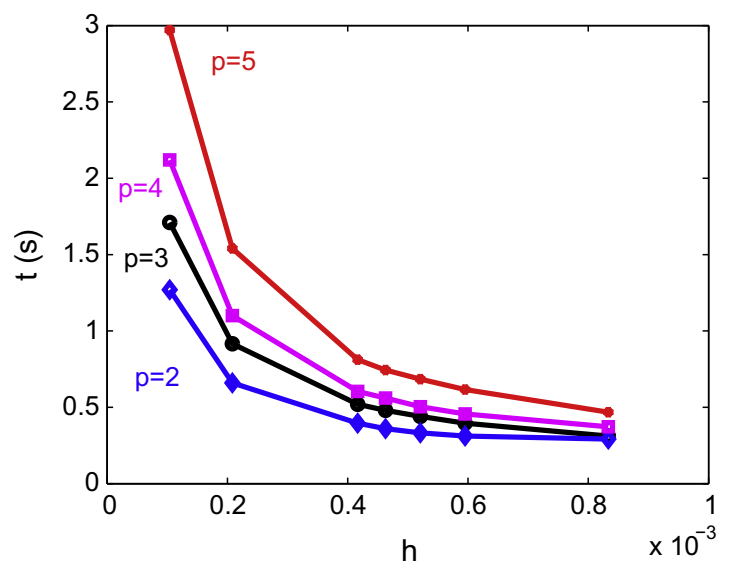

Fig. 3. Timing runs as a function of the inverse of the number of intervals for a field line on the flux surface corresponding to $s=0.526$.

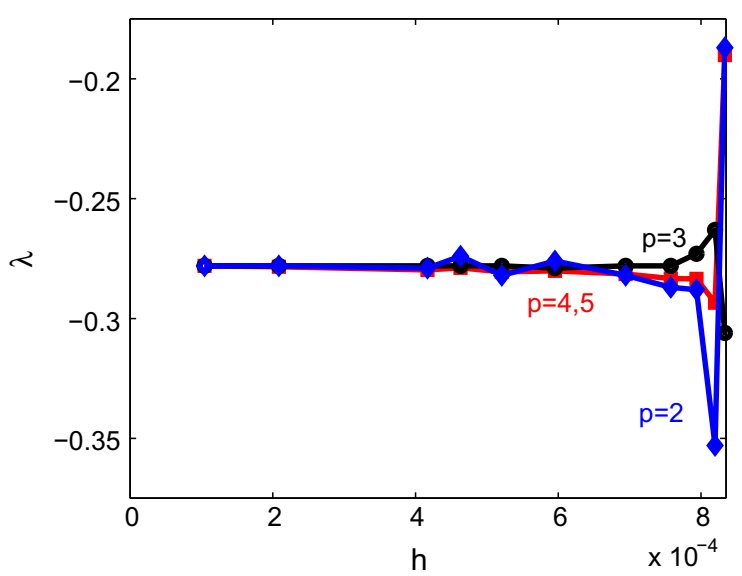

Fig. 4. Eigenvalue convergence plot for different basis function polynomial order as a function of the inverse of the number of intervals for a field line on the flux surface corresponding to $s=0.526$.

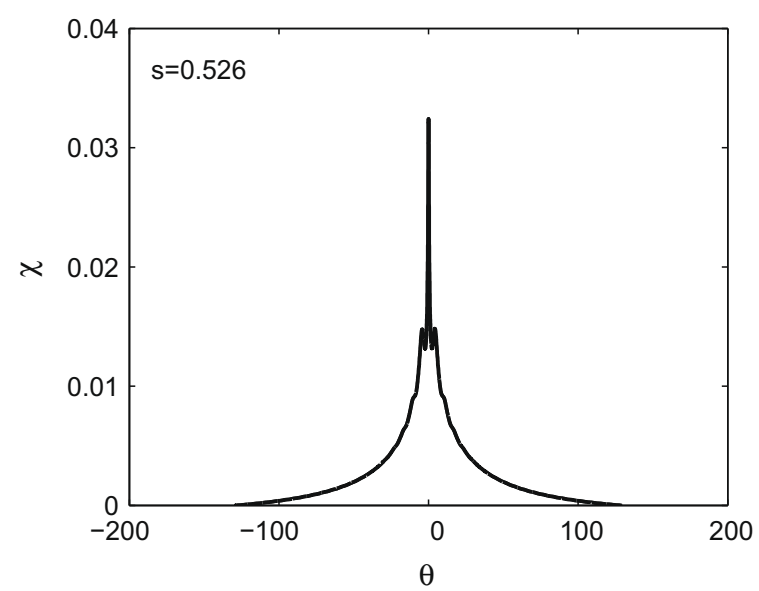

Fig. 5. Converged eigenfunction along a magnetic field line where the label $\chi$ corresponds to the perturbed function $Y$ on a the flux surface with $s=0.526$.

that for the case $p=3$, it was convenient set the value of $\zeta_{1}=0$. Fig. 5 shows the converged eigenfunction along a field line portraying a typical ballooning structure.

\section{Summary}

The incompressible ideal ballooning mode equation is broken into a variable number of intervals using the COOL finite element discretization scheme. This replaces the poloidal angle with an index that labels the interval and a variable that rep- 
resents location within the interval and corresponds to a value between negative unity and unity. The perturbation function is approximated by a series of basis functions, comprised of variable order Legendre polynomials, multiplied by perturbation elements. This reduces the ballooning mode equation to a special block pentadiagonal matrix equation. The equation is then solved using an inverse vector iteration method in the subroutine PAMERA. One major advantage of the formulation we have implemented with the COOL finite element scheme is that we can alter the order of the polynomial basis functions to best adapt it to the problem at hand. More specifically, we can vary the order of the polynomial from linear to quadratic, or cubic, or in fact arbitrary order without any reprogramming of the code. The order $p$ of the Legendre polynomial used to construct the basis functions $h_{i}$ shown in Eq. (4) constitutes an input parameter.

Numerical computations reveal that BECOOL recovers precisely the eigenvalues calculated by VVBAL when using second order Legendre polynomials. Further tests portray a time-based need to find an optimal Legendre polynomial order for intensive computations. For computational efficiency, there is usually a tradeoff between mesh size and the order of the polynomial applied. It is determined that the ballooning mode equation discretized with cubic Legendre polynomials appears to be the optimal case to consider because the imposition of higher order elements for the incompressible ballooning problem becomes more time consuming without increasing the accuracy of the computed eigenvalue as demonstrated in Figs. 3 and 4.

The COOL method is currently being adapted to the more complicated global MHD stability problem through the TERPSICHORE code [13] which is described by a set of partial differential equations rather than the second order ordinary differential equation that is derived to model ballooning modes. Therefore, the BECOOL code development offers very useful insights on the critical properties and problems associated with the COOL discretization technique.

\section{Acknowledgments}

I (GAC) would like to thank the anonymous donor alumni from the University of the South who supported this research. This research was also partially sponsored by the Fonds National Suisse de la Recherche Scientifique and Euratom. We thank S.P. Hirshman for the use of his VMEC code.

\section{References}

[1] J.W. Connor, R.J. Hastie, J.B. Taylor, High mode number stability of an axisymmetric toroidal plasma, Proc. Roy. Soc. London Ser. A 365 (1979) 1-17.

[2] R.L. Dewar, A.H. Glasser, Ballooning mode spectrum in general toroidal systems, Phys. Fluids 26 (1983) 3038 -3052.

[3] D. Correa-Restrepo, Ballooning modes in three-dimensional MHD equilibria with shear, Z. Naturforsch 33a (1978) 789-791.

[4] J. Nührenberg, R. Zille, Equilibrium and stability of low-shear stellarators, in: Proc. Joint Varenna Lausanne Int. Conf. on Theory of Fusion Plasmas, EUR11336EN, 1987, pp. 3-23.

[5] W.A. Cooper, D.B. Singleton, R.L. Dewar, Spectrum of ballooning instabilities in a stellarator, Phys. Plasmas 3 (1996) 275-280 (Erratum Phys. Plasmas 3 (1996) 3520).

[6] W.A. Cooper, Variational formulation of the linear MHD stability of 3D plasmas with noninteracting hot electrons, Plasma Phys. Contr. Fusion 34 (1992) 1011-1036.

[7] R. Sanchez, J.A. Jimenez, L. Garcia, A. Varias, Compressibility effects on ideal and resistive ballooning stability in the TJ-II heliac device, Nucl. Fusion 37 (1997) 1363-1373.

[8] N. Nakajima, High-mode-number ballooning modes in a heliotron/torsatron system. II. Stability, Phys. Plasmas 3 (1996) $4556-4567$.

[9] R. Sanchez, S.P. Hirshman, J.C. Whitson, A.S. Ware, COBRA: an optimized code for fast analysis of ideal ballooning stability of three-dimensional magnetic equilibria, J. Comput. Phys. 161 (2000) 576-588.

[10] A. Ahusborde, R. Gruber, M. Azaiez, M.L. Sawley, Physics-conforming constraints-oriented numerical method, Phys. Rev. E 75 (2007) 056704.

[11] K. Appert, M. Azaiez, R. Gruber, Modes of a plasma-filled waveguide determined by a numerical hp method, Commun. Comput. Phys. 5 (2009) 413425.

[12] R. Gruber, W.A. Cooper, M. Beniston, M. Gengler, S. Merazzi, Software development strategies for parallel computer architectures, Phys. Rep. 207 (1991) 167-214.

[13] D.V. Anderson, W.A. Cooper, R. Gruber, S. Merazzi, U. Schwenn, Methods for the efficient calculation of the (MHD) magnetohydrodynamic stability properties of magnetically confined fusion plasmas, Int. J. Supercomp. Appl. 4 (1990) 34-47.

[14] R. Gruber, J. Rappaz, Finite Element Methods in Linear Ideal MHD, Springer Series in Computational Physics, Springer-Verlag, Berlin, 1985.

[15] A.N. Lowan, N. Davids, A. Levenson, Table of the zeros of the Legendre polynomials of order $1-16$ and the weight coefficients for Gauss' mechanical quadrature rule, Bull. Am. Math. Soc. 48 (1942) 739-742.

[16] S.P. Hirshman, W.I. Van Rij, P. Merkel, Three-dimensional free boundary calculations using a spectral Green's function method, Comput. Phys. Commun. 43 (1986) 143-155.

[17] Y. Nakamura, T. Matsumoto, M. Wakatani, S.A. Galkin, V.V. Drozdov, A.A. Martynov, Yu.Yu. Poshekhonov, K. Ichiguchi, L. Garcia, B.A. Carreras, C. Nührenberg (née Schwab), W.A. Cooper, J.L. Johnson, Comparison of the calculations of the stability properties of a specific stellarator equilibrium with different MHD stability codes, J. Comput. Phys. 128 (1996) 43-57.

[18] A.H. Boozer, Guiding center drift equations, Phys. Fluids 23 (1980) 904-908. 